\title{
Ecologie des Leishmanioses dans le sud de la France
}

\author{
5. - Pouvoir infestant comparé des diverses formes \\ de Leishmaniose canine vis-à-vis de Pblebotomus ariasi \\ Tonnoir, 1921.
}

\author{
par J.-A. RIOUX, G. LANOTTE, H. CROSET et J.-P. DEDET \\ (collaboration technique: A. Martini-Dumas) \\ Laboratoire d'Ecologie médicale et de Pathologie parasitaire ( $\mathrm{P}^{r} \mathrm{~J} .-\mathrm{A}$. Rioux), \\ Faculté de Médecine, F 34000 Montpellier \\ et Station expérimentale, F 34000 La Borie Nouvelle
}

\begin{abstract}
Résumé
En complément de leur enquête écologique sur l'endémie leishmanienne en France, les auteurs étudient le pouvoir infestant des diverses formes cliniques de Leishmaniose canine vis-à-vis du vecteur habituel, Phlebotomus ariasi Tonnoir, 1921.

Les chiens leishmaniens en phase infra-olinique ne sont que très faiblement contagieux alors qu'en période d'état ils infestent le vecteur dans les proportions de 70 à $80 \%$. Les animaux traités par les Diamidines conservent intact leur pouvoir infestant, tout au moins dans les premières semaines qui suivent la fin de la cure. Par ailleurs, le calcul de la densité des chiens infestants $\left(0,09 \mathrm{~km}^{2}\right)$ dans un microfoyer des Cévennes méridionales montre que la distance entre deux animaux atteints est suffisamment faible $(3,3 \mathrm{~km})$ pour expliquer la circulation de l'affection par le seul déplacement actif du réservoir domestique.
\end{abstract}

\section{Summary}

In addition to their ecological investigation about leishmaniasis in the South of France, the authors study the infectivity of dogs at various clinical phases to Phlebotomus ariasi Tonnoir, 1921 , the usual vector for this infection in the region. 
Leishmanial dogs are only slightly infective when they are in the sub-clinical phase; they infect, on the contrary, a great proportion $(70$ to $80 \%$ ) of the vector population when they are in a clinical phase. The animals treated with Diamidines remain infective for at least the first weeks after.

On the other hand, the density of infected dogs $\left(0,09 / \mathrm{km}^{2}\right)$ in a micro-focus in the southern Cevennes, shows that the distance between two animals infected with leishmaniasis is short enough $(3,3 \mathrm{~km})$ to explain the possibility of transmission by the active movements of the domestic reservoir alone.

Une récente enquête, basée sur l'application des techniques d'immunofluorescence nous a permis de dresser un premier bilan de l'enzootie leishmanienne en Cévennes. Ainsi, l'examen de 1387 sérums de chiens, prélevés le long d'un transect Sud-Nord de 65 kilomètres, a montré qu'au centre du foyer, le taux de positivité n'excédait qu'exceptionnellement $5 \%$ (Lanotte et coll., 1970). Au surplus, parmi les cas dépistés, une proportion non négligeable concernait des formes infra-cliniques ou tout au moins à très faible expression cutanée ou générale. Dans ces derniers cas, seule l'adénoculture révélait le parasite.

Dès lors, il nous a paru intéressant de comparer le pouvoir infestant des diverses formes cliniques vis-à-vis du vecteur habituel, Phlebotomus ariasi Tonnoir, 1921, de manière à déterminer l'incidence leishmanienne minimale, c'est-à-dire, pour un foyer donné, le taux de chiens infestants nécessaire et suffisant au maintien de l'enzootie.

\section{A. - Matériel et méthodes}

\section{I. - Choix des chiens leishmaniens.}

Les contraintes techniques inhérentes à une telle étude ne nous ont pas permis de réaliser notre expérimentation sur un nombre important d'animaux. Aussi avons-nous été amené à sélectionner les formes cliniques les plus typiques, de manière à obtenir un échantillonnage représentatif des divers stades évolutifs. Cinq chiens, répondant à ces critères ont été choisis : trois étaient atteints de Leishmaniose clinique, dont deux récemment traités par les Diamidines; les deux autres présentaient une forme asymptomatique non traitée.

CHIEN N 3729 (Lucky). Croisé Groenendael, ơ, 2 ans. Chien de garde, originaire de Lugné (Hérault). Né de mère leishmanienne (Olga, cf. ci-après). Premiers signes apparus en septembre 1970: dépilation en placard du dos et du siège. Examiné le 29 janvier 1971: amaigrissement intense ; fontes des crotaphytes et des muscles fessiers ; kératite légère ; furfur des oreilles et des régions périoculaires. Immunofluorescence : 1/2560 Fixation du complément : sérum anticomplémentaire. Adénoculture positive. Le 20-4-1971: aggravation clinique. 
Immunofluorescence: 1/5120. Fixation du complément: sérum anticomplémentaire. Le 9-7-1971: signes cutanés en discrète régression mais profonde altération de l'état général; parésie du train postérieur.

Diagnostic: Leishmaniose en période terminale avec altération profonde de l'état général, signes cutanés et parésie du train postérieur.

Le 10-7-1971: placé sous moustiquaire pour infestation de Phlebotomus ariasi.

CHIEN N 3685 (Peter). Teckel, ơ , 4 ans. Chien de compagnie, originaire de Ganges (Hérault). Début de la maladie en octobre 1970; Pityriasis discret du dos et de la tête. En décembre de la même année, signes cutanés plus importants: furfur et dépilation généralisés; plaques érosives du dos et de la face. En février 1971 apparition de nouveaux signes: fonte des crotaphytes: kératite discrète ; polyadénapathie. Immunofluorescence : 1/2560. Fixation du complément: sérum anticomplémentaire. Adénoculture positive. Du 11-12 au 19-12 1970, du 6-2 au 13-2-1971 et du 10-8 au 17-8-1971: traitement par les Diamidines. Malgré ces trois cures l'état général reste alarmant: apparition d'escarres sur le siège, les épaules et le poitrail ; lésions érosives de la truffe et des oreilles; kérato-conjonctivite intense.

Diagnostic: Leishmaniose gravissime d'évolution continue, résistant aux Diamidines.

Le 17-8-1971, placé sous moustiquaire pour infestation de Phlebotomus ariasi.

CHIEn N $\mathrm{N}^{\circ} 3682$ (Olga). Croisé Groenendael, +5 ans. Chien de garde, originaire de Lugné (Hérault). Début des symptômes en mai 1970. Dépilation généralisée. Examiné le 18-6-1970 : Leucoformol-gélification positive. Traitement par les Diamidines. Le 20-7-1970: état général conservé mais pityriasis diffus du dos et des lombes; dépilation en lunettes; lésion végétante de la truffe; kératite bilatérale. Immunofluorescence: 1/2560. Fixation du complément : sérum anti-complémentaire. Adénoculture positive.

Diagnostic: Leishmaniose en période d'état avec prédominance des signes cutanéomuqueux.

Le 22-7-1970, placé sous moustiquaire pour infestation de Phlebotomus ariasi.

CHIEN N 3727 (Franca). Epagneul breton, đo, 4 ans. Originaire de Pont-d'Hérault (Gard), mais déplacé à Ganges dès la naissance. Leishmaniose découverte le 30-3-1971 à l'occasion d'un dépistage systématique. Immunofluorescence: 1/160. Fixation du complément: 1/40. Le 23-6-1971, nouvel examen: absence de signes cutanés ou oculaires; excellent état général. Immunofluorescence: 1/2560. Fixation du ocmplément: 1/320. Adénoculture positive. Le 16-8-1971: discrète lésion érosive de la patte arrière gauche; état général conservé. Immunofluorescence: $1 / 160$. Fixation du complément: $1 / 80$.

Diagnostic: Leishmaniose paucisymptomatique en période initiale.

Le 17-7-1971, placé sous moustiquaire pour infestation de Phlebotomus ariasi.

CHIEN N 3690 (Tutur). Croisé Epagneul, $\uparrow, 3$ ans. Chien de berger, originaire d'Esparon (Gard). Leishmaniose découverte le 1-12-1970 à l'occasion d'un dépistage systématique: immunofluorescence: $1 / 160^{\circ}$. Absence de signes cutanés et oculaires, excellent état général. Le 15-12-1970: adénoculture négative. Le 2-6-1971: état clinique inchangé à l'exception d'un discret intertrigo de la patte antérieure droite. Immunofluorescence : $1 / 640^{\circ}$.

Diagnostic: Leishmaniose asymptomatique.

Le 13-7-1971, placé sous moustiquaire pour infestation de Phlebotomus ariasi. 


\section{II. - Infestation expérimentale de Phlebotomus ariasi Tonnoir, 1921.}

Pour mener à bien une telle étude, nous avons utilisé une technique simple et rapide permettant d'obtenir in natura un nombre important de Phlébotomes gorgés. Nous rappelons l'essentiel des manipulations qui, pour la plupart, ont été réalisées en juillet-août dans la station expérimentale de La Borie-Nouvelle (Hérault).

Les Phlébotomes, capturés à l'extérieur, contre les murs, sont introduits, par lots de 500 à 1.000 , dans une série de phlébotomaires tendues sous abris (caves ou resserres). Chaque phlébotomaire renferme un chien leishmanien placé dans une cage métallique à larges mailles. Le lendemain, les Phlébotomes gorgés sont retirés à l'aide d'un aspirateur-nasse et introduits extemporanément dans des pots à fond de plâtre (cf. J.-A. Rioux et coll., 1969). Chaque pot reçoit en moyenne 25 individus. Les pots sont ramenés au laboratoire et conditionnés en insectarium à $23^{\circ}$. Un lot non gorgé ou nourri sur chien sain sert de témoin.

Les Phlébotomes sont disséqués entre le $7^{\mathrm{e}}$ et le $9^{\mathrm{e}}$ jour. Pour ce faire, les femelles sont capturées individuellement à l'aide d'un tube de verre, anesthésiées au $\mathrm{CO}_{2}$ et déposées dans une goutte d'eau salée à $0,6 \%$. Une aiguille montée, appliquée sur l'une des faces latérales $\mathrm{du}$ thorax maintient l'insecte sur la lame pendant qu'une deuxième tire sur les deux derniers segments abdominaux, de manière à les détacher et à entraîner avec eux le tube digestif. Une traction lente et continue, rigoureusement dirigée dans l'axe du corps, permet de réaliser de très belles dissections comportant à la fois l'œsophage, le jabot, l'intestin moyen et l'intestin postérieur. La préparation, recouverte d'une lamelle, est examinée à fort grossissement. Les parasites sont surtout visibles dans le segment antérieur de l'intestin moyen, immédiatement en arrière du sphincter œsophagien. L'existence d'un bol alimentaire incomplètement digéré oblige parfois à sectionner la paroi intestinale, de façon à libérer d'éventuelles formes promastigotes, camouflées dans la masse sanguine. Enfin, l'examen des follicules ovariens permet de déterminer le stade du cycle trophogonique.

\section{B. - Résultats}

Les résultats, exprimés dans le tableau I, montrent les nettes différences de pouvoir infestant selon l'état clinique : dans les formes asymptomatiques et paucisymptomatiques ce pouvoir est faible sinon nul ( $n^{\circ} 3690$ et 3727). Par contre, dès l'apparition des signes cutanés et avant toute atteinte de l'état général, l'animal infeste massivement le vecteur. Au surplus, le traitement récent par les Diamidines n'entraîne aucune modification du pouvoir de multiplication des formes promastigotes $\left(\mathrm{n}^{\circ} 3682,3685\right.$ et 3729 ).

De tels résultats confirment les travaux de L. Deane (1956) et S. Adler et O. Theodor (1932 et 1935). Ces derniers auteurs ont en effet montré que le chien leishmanien en phase d'état infestait Phlebotomus perniciosus Newstead, 1911, dans des proportions de $32 \%$ à $65,4 \%$. De même, deux chiens leishmaniens testés à trois reprises, depuis le début de l'évolution jusqu'à la période d'état, infestaient le vecteur dans des proportions progressivement croissantes de 0 à $79 \%$. 


\section{Tableau I}

\begin{tabular}{|c|c|c|c|c|}
\hline Chiens & Formes cliniques & $\begin{array}{c}\text { Nombre } \\
\text { de Phlébo- } \\
\text { tomes gorgés } \\
(P . \text { ariasi })\end{array}$ & $\begin{array}{l}\text { Nombre } \\
\text { de Phlébo- } \\
\text { tomes } \\
\text { infestés }\end{array}$ & $\begin{array}{c}\% \\
\text { d'infestation }\end{array}$ \\
\hline $\begin{array}{l}\mathrm{n}^{\circ} 3690 \\
\text { (Tutur) } \ldots\end{array}$ & asymptomatique, non traitée & 66 & 0 & $0 \%$ \\
\hline $\begin{array}{l}\mathrm{n}^{\circ} 3727 \\
\text { (Franca) }\end{array}$ & $\begin{array}{l}\text { forme de début, paucisymp- } \\
\text { tomatique non traitée. }\end{array}$ & 63 & 7 & $11 \%$ \\
\hline $\begin{array}{l}\mathrm{n}^{\circ} 3682 \\
(\text { Olga })\end{array}$ & $\begin{array}{l}\text { période d'état ; traitée (Dia- } \\
\text { midines) }\end{array}$ & 61 & 44 & $72 \%$ \\
\hline $\begin{array}{l}\mathrm{n}^{\circ} 3685 \\
\text { (Peter) } \ldots\end{array}$ & $\begin{array}{l}\text { ṕ́riode terminale; traite- } \\
\text { ment récent (Diamidines) }\end{array}$ & 89 & 72 & $81 \%$ \\
\hline $\begin{array}{l}\mathrm{n}^{\circ} 3729 \\
\text { (Lucky) }\end{array}$ & $\begin{array}{l}\text { période terminale; traite- } \\
\text { ment récent (Diamidines) }\end{array}$ & 73 & 61 & $83 \%$ \\
\hline
\end{tabular}

Des observations précédentes, il résulte que :

$1^{\circ}$ ) Un chien leishmanien en période initiale n'est que très faiblement infestant, alors que, dans le même temps, le taux d'anticorps circulants est significatif et le parasite présent dans les ganglions. Rappelons que cette phase peut durer plusieurs mois sinon plusieurs années.

$2^{\circ}$ ) Un chien leishmanien, traité par les Diamidines, conserve intact son pouvoir infestant vis-à-vis de Phlebotomus ariasi, tout au moins dans les premières semaines qui suivent la fin de cure. A ce titre, il doit être considéré comme contagieux et partant, soustrait à la piqûre des Phlébotomes. De même, il est prudent de l'écarter du voisinage des enfants.

$3^{\circ}$ ) Dans le foyer leishmanien des Cévennes, le nombre de chiens infestants reste toujours très inférieur au nombre total d'animaux atteints. Ainsi dans la zone enzootique de Ganges (Hérault ; $160 \mathrm{~km}^{2}$ ), le taux de chiens infestés (incidence «globale») avoisine $4,6 \%$ contre $2,3 \%$ pour celui des formes cliniquement exprimées (incidence \& utile»). Ce dernier chiffre, relativement bas, pose le problème du taux de réservoirs effectifs, nécessaire et suffisant à l'entretien des foyers. Or, rapporté à l'unité de surface il correspond à 0,09 chien infestant au km² c'est-à-dire 1 chien infestant pour $11 \mathrm{~km}^{2}$. 
En d'autres termes, dans l'hypothèse d'une distribution régulière (1) de la Leishmaniose, deux chiens leishmaniens sont séparés par un intervalle de $3,3 \mathrm{~km}$, distance facilement couverte au cours des déplacements. Dans ces conditions, il n'est nul besoin d'invoquer le transport passif d'animaux malades (changement de propriétaires, vacances, chasse, etc.) pour expliquer la circulation de l'affection tout au moins dans les secteurs les plus contaminés.

Tableau II. - Infestation de Phlebotomus perniciosus Newst., 1911 (d'après S. Adler et O. Theodor, 1932).

\begin{tabular}{|c|c|c|}
\hline Ohiens & $\begin{array}{c}\text { Nombre } \\
\text { de Phlébotomes gorgés }\end{array}$ & $\%$ d'infestatior \\
\hline $\mathrm{n}^{\circ} 1 \ldots \ldots \ldots \ldots \ldots \ldots \ldots$ & 42 & $62,5 \%$ \\
\hline $\mathrm{n}^{\circ} 2 \ldots \ldots \ldots \ldots \ldots \ldots$ & 26 & $65,4 \%$ \\
\hline $\mathrm{n}^{\circ} 3 \ldots \ldots \ldots \ldots \ldots \ldots \ldots$ & 36 & 32 \\
\hline
\end{tabular}

Tableau III. - Evolution du pouvoir infestant des deux chiens leishmaniens vis-à-vis de Phlebotomus perniciosus Newst., 1911 (d'après S. Adler et O. Theodor, 1935).

\begin{tabular}{|c|c|c|c|}
\hline Chiens & Mai-juin & Juillet-août & Septembre-octobre \\
\hline $\mathrm{n}^{\circ} 3 \ldots \ldots \ldots \ldots \ldots$ & $15 \%$ & $31 \%$ & $79 \%$ \\
\hline $\mathrm{n}^{\circ} 8 \ldots \ldots \ldots \ldots \ldots$ & $0 \%$ & $23 \%$ & $71 \%$ \\
\hline
\end{tabular}

\section{Bibliographie}

AdLer (S.) et Theodor (O.) 1932. - Investigations on mediterranean Kala-azar, VI. Canine visceral leishmaniasis. Proc. Roy. Soc. London, 110: 402-412.

- et -, 1935. - Investigations on mediterranean Kala-azar. VH. Further observations on canine visceral leishmaniasis. Proc. Roy. Soc., 116: 494-505

— et -, 1935. - Investigations on mediterranean Kala-azar. IX. Feeding experiments with Phlebotomus perniciosus and other species on animals infected with Leishmania infantum. Proc. Roy. Soc. London, 116: 516-542.

(1) En fait, la distribution de la Leishmaniose canine correspond plutôt au type «contagieux » que \& régulier ». 
Christophers (S. R.) et ShortT (H. E.), 1925. - The development of the parasite of Indian Kala-azar in the sandfly Phlebotomus argentipes, Annandale and Brunetti. Ind. J. Med. Res., 12 : 605-607.

DEANE (L. M.), 1956. - Leishmaniose visceral no Brasil. Estudos sobre reservatories e transmissores realizados no estado do Ceara. Serviço Nacional de Educaçao Sanitaria éd. Rio de Janeiro, 162 p.

Lanotte (G.), Rioux (J.-A.), Dedet (J.-P.) et Martini-Dumas (A.), 1970. - Utilisation des méthodes de fixation du complément et d'immunofluorescence dans le dépistage de la leishmaniose canine. Soc. Fr. de Paras. (séance du 10 mai 1971).

Rioux (J.-A.), Croset (H.), Aboulker (J.-P.) et Papierok (B.), 1972. - Ecologie des Leishmanioses dans le sud de la France. 4. Infestation d'une population naturelle de Phlebotomus ariasi, Tonnoir, 1921, Ann. Paras. Hum. Comp. 47 (sous presse).

-, Golvan (Y.-J.), Croset (H.), Tour (S.), houin (R.), Abonnenc (E.), Petit-Didier (M.), Volhardt (Y.), Dedet (J.-P.), Albaret (J.-L.), Lanotte (G.) et Quilici (M.), 1969. - Epidémiologie des leishmanioses dans le sud de la France. Monographie de l'Inst. Nat. Sant. Rech. Méd., $\mathrm{n}^{\circ} 37: 223$ p.

—, Lanotte (G.), Croset (H.), Houin (R.), Guy (Y.), et Deder (J.-P.), 1972. - Ecologie des Leishmanioses dans le sud de la France - 3. Réceptivité comparée de Phlebotomus ariasi, Tonnoir, 1971 et Rhipicephalus turanicus Pomerancev et Matikasvili, 1940, vis-à-vis de Leishmania donovani (Laveran et Mesnil, 1903). Ann. Paras. Hum. Comp., 47 : 147-157.

\title{
RECTIFICATIF
}

\section{Ecologie des Leishmanioses dans le sud de la France}

3. - Réceptivité comparée de Phlebotomus ariasi Tonnoir, 1921

et Rhipicephalus turanicus Pomerancev et Matikasvili, 1940 vis-à-vis de Leishmania donovani (Laveran et Mesnil, 1903)

\author{
par J.-A. Rioux, G. Lanotte, H. Croset, R. Houin, Y. Guy et J.-P. Dedet
}

(Collaboration technique: A. BRÈs et A. Martini-Dumas)

Nous informons les lecteurs que dans l'article ci-dessus rappelé, paru dans le $\mathrm{n}^{\circ} 1 / 1972$, pp. 147 157 , les légendes des figures 2 et 3 se trouvent inversées. De plus, dans ces mêmes légendes, il faut lire hôte à la place de note. 